\title{
Metodologías activas en la enseñanza de las ciencias: revisión y análisis de publicaciones en revistas del área enseñanza en la década de 2008 a 2018
}

Roberta Cristina Moreira Simões rcmsimoes@hotmail.com orcid.org/0000-0001-5503-9269 Instituto Federal de Educação, Ciência e Tecnologia do Rio de Janeiro (IFRJ), Nilópolis, Rio de Janeiro, Brasil

\section{Ana Lúcia Rodrigues Gama}

Russo

nalurusso@gmail.com

orcid.org/0000-0002-9763-3909

Instituto Federal de Educação, Ciência e Tecnologia do Rio de Janeiro (IFRJ), Duque de Caxias e Nilópolis, Rio de Janeiro, Brasil

Eduardo dos Santos de Oliveira Braga

obeduardobraga@hotmail.com

orcid.org/0000-0002-8742-6981

Instituto Federal de Educação, Ciência Tecnologia do Rio de Janeiro (IFRJ), Duque de Caxias e Nilópolis, Rio de Janeiro, Brasil

Giselle Rôças de Souza Fonseca giselle.rocas@ifri.edu.br orcid.org/0000-0002-1669-7725 Instituto Federal de Educação, Ciência e Tecnologia do Rio de Janeiro (IFRJ), Nilópolis e Rio de Janeiro, Rio de Janeiro, Brasil

\section{RESUMEN}

Este artículo presenta una búsqueda de artículos científicos sobre metodologías activas publicados en revistas brasileñas Qualis A1 del Área Enseñanza de la Coordenação de Aperfeiçoamento de Pessoal de Nível Superior (CAPES). Para responder a la pregunta: ¿Cómo ha sido utilizado y discutido el tema de metodologías activas por los profesores y los investigadores de la enseñanza de las Ciencias?, el trabajo tiene como objetivo realizar la revisión de los artículos relacionados a la enseñanza de las Ciencias en el período de 2008 a 2018. Los objetivos específicos incluyen seleccionar publicaciones y revisar los contextos de la enseñanza en que las estrategias son desarrolladas, sin la pretensión de evaluar o disertar sobre todas las estrategias de metodologías activas disponibles. El trabajo adoptó un enfoque cualitativo, constituyéndose como una investigación bibliográfica en el ámbito del Estado del Conocimiento. Las revistas nacionales fueron seleccionadas consultando el portal CAPES, en la plataforma Sucupira, utilizando los filtros: Área de Enseñanza y cuatrienio 2013-2016; y para la selección de artículos, se definieron palabras claves relacionadas con las metodologías activas. Como resultado, diecinueve revistas y un total de treinta y nueve publicaciones fueron seleccionadas. Las metodologías activas más frecuentes en los artículos son Enseñanza por Problemas y Enseñanza por Investigación, y los niveles de educación más sobresalientes son la Educación Superior y la Escuela Secundaria. En el marco temporal de este trabajo hubo un aumento en el número de publicaciones de 2015 a 2018. Llegamos a la conclusión de que los profesores y los investigadores han estado discutiendo metodologías activas, utilizando diferentes estrategias, en diferentes niveles de educación. Se destaca la importancia de la profundización teórica para el desarrollo del trabajo basado en el uso de metodologías activas, evitando un mal uso, o uso sin control, como un desorden metodológico.

PALABRAS-CLAVE: Metodologías Activas. Enseñanza de las Ciencias. Estado del Conocimiento. Investigación Bibliográfica. 


\section{INTRODUCCIÓN}

En los procesos de enseñanza y aprendizaje, la información obtenida por los estudiantes debe articularse, contextualizarse y transformarse en conocimiento, permitiendo al estudiante ejercer la ciudadanía. Sin embargo, extrapolar el acceso a la información, convirtiéndola en conocimiento, requiere la participación activa de todos los involucrados en los procesos y el incentivo para que los estudiantes se conviertan en más que reproductores de información y observadores del mundo (BERBEL, 2011).

En este contexto, las metodologías activas (MA) se han utilizado en varios niveles de educación en Brasil en busca de una participación más activa de los estudiantes durante el aprendizaje (MORAN, 2018). Sin embargo, a pesar de que las MA están actualmente en uso, los profesores y los estudiantes no siempre reconocen sus roles en los procesos de enseñanza y aprendizaje, por lo que es esencial profundizar el enfoque metodológico elegido para aplicarlo de la manera más adecuada.

La historia de las MA en la enseñanza tiene origen en el final del siglo XIX con el Movimiento Progresista en la Educación, también denominado Escuela Nueva, cuyos ideales eran contrarios al modelo tradicional de enseñanza vigente en la época. Dewey (1950) fue uno de los representantes de estas ideas y defendió prácticas de enseñanza centradas en el estudiante como protagonista de su aprendizaje, con la participación activa por medio de la reflexión y de la problematización de la realidad. La Escuela Nueva inspiró al Aprendizaje Basado en Problemas y en Proyectos, y otras propuestas fueron desarrolladas a partir de los principios pautados en esta teoría (SOUZA; DOURADO, 2015; ZOMPERO; LABURÚ, 2011).

En este trabajo no tenemos la intención de realizar un análisis conceptual profundo de todas las estrategias con MA disponibles, o la profundización metodológica en cada abordaje, sino presentar una breve exposición y definición de algunas posibilidades de MA que más se destacaron en el análisis de los artículos. Las MA pueden desarrollarse a partir de diferentes estrategias, algunas de las cuales se describen brevemente a continuación.

La Enseñanza por Investigación se caracteriza por la aplicación de actividades de investigación que incluyen resolución de problemas, la formulación de hipótesis, la planificación de estudios, la búsqueda de información, el análisis e interpretación de datos y la posterior comunicación de los resultados al grupo (ZOMPERO; LABURÚ, 2011). Para Carvalho (2013), las secuencias de la Enseñanza por Investigación permiten la contextualización de problemas y la discusión de ideas a partir de conocimientos previos, lo que lleva a los estudiantes al desarrollo de un lenguaje científico en la construcción del conocimiento.

El Aprendizaje Basado en Problemas (ABP) (Problem-based learning) y la Metodología de Problematización con apoyo del arco de Maguerez (MP) se producen a partir de la resolución de problemas, sin embargo, con diferentes enfoques. En la ABP las asignaturas están integradas y hay una reorganización del currículo. Los pasos incluyen la identificación de problemas, la formulación de hipótesis, la investigación y aplicación de conocimientos para resolver problemas. La MP no requiere una organización curricular amplia e incluye las siguientes etapas: Observación de la realidad; Establecimiento de puntos claves, donde son 
identificadas las posibles causas de los problemas y su relación con la realidad social; Teorización, donde tiene lugar la investigación; Preparación de hipótesis de solución; y Aplicación a la realidad, que implica la toma de decisiones para resolver el problema con base en una intervención directa en la realidad social (BERBEL, 2011).

El Estudio de Caso (Teaching case) se caracteriza por la resolución de problemas que involucran la realidad, presentándose a través de casos de investigación en narraciones cortas que involucran diálogos y buscan despertar el interés y la curiosidad de los estudiantes, llevándolos a una decisión sobre cómo resolver el problema (QUEIROZ; CABRAL, 2016).

En el Aula Invertida (Flipped classroom), los estudiantes usan contenidos estudiados antes de la clase para actividades orientadas por el profesor, como interacción con sus compañeros que incluye resolución de problemas, investigación, desafíos y desarrollo de proyectos. El profesor pone a disposición actividades previas on-line, verifica las dudas y planifica las próximas clases a partir de las dificultades presentadas, utilizando herramientas digitales (VALENTE, 2014b).

Finalmente, Eric Mazur, de la Universidad de Harvard, desarrolló la Instrucción por Pares (Peer Instruction), un tipo de Aula Invertida, con el objetivo de mejorar el aprendizaje conceptual de los estudiantes universitarios de Física. El profesor planifica los contenidos, coloca a disposición textos y preguntas en línea e identifica posibles dudas. Durante las clases, hay momentos intercalados de exposición de conceptos por parte del profesor y aplicación de preguntas, fomentando discusiones entre los estudiantes. Para evaluar las preguntas se utilizan dispositivos electrónicos o tarjetas. En las preguntas previas a la prueba, hay tres posibilidades con respecto al porcentaje total de respuestas correctas: por debajo del $30 \%$, el profesor explica nuevamente los conceptos; entre $30 \%$ y $70 \%$ se discuten las preguntas con los pares y se realizan preguntas post-prueba para verificar posibles mejoras en el total de respuestas correctas; y por encima del $70 \%$, el profesor explica un nuevo concepto. Al final de cada pregunta post-prueba, el profesor explica las preguntas y responde las dudas (MAZUR, 2015; ARAUJO; MAZUR, 2013).

Los libros, cursos y conferencias que invitan a los profesores a utilizar metodologías pedagógicas no convencionales durante las clases, entre ellas MA, nos llevan a algunas reflexiones. Primero, no siempre el profesor conoce con cierta profundidad los principios de la metodología elegida (SILVA, 2011); segundo, hay un desajuste entre los sistemas de evaluación y la propuesta metodológica (BERGMANN; SAMS, 2016); tercero, porque al buscar atender todos los contenidos previstos en el plan de estudios, la metodología no siempre se desarrolla adecuadamente (MORAN, 2018); y finalmente, si habrá continuidad en el desarrollo de metodologías y métodos en los próximos períodos con los estudiantes, para no caer en un uso fragmentado de la estrategia propuesta BELTRÃO et al., 2010; LACANALLO et al., 2007).

Con base en los elementos presentados anteriormente, y considerando la diversidad de las MA, se plantearon algunas preguntas: ¿Cuánta tendencia hay en el uso de las MA por parte de los profesores de Ciencias? ¿Los profesores que eligen trabajar con MA son claros y/o están listos para trabajar con la MA? 
Finalmente, nos preguntamos: ¿Cómo ha sido utilizado y discutido el tema de MA por los profesores y los investigadores de la enseñanza de las Ciencias? Para este estudio, elegimos responder a la tercera y última pregunta, con base en revisiones bibliográficas del carácter del Estado del Conocimiento. Por lo tanto, asumimos como objetivo general realizar una revisión de las investigaciones publicadas en artículos científicos que aborden las MA en enseñanza de Ciencias, basada en el análisis de publicaciones indexadas por Qualis de la CAPES. Los objetivos específicos incluyen seleccionar publicaciones y revisar los contextos de enseñanza en que las MA son desarrolladas, como las estrategias abordadas en los artículos, en los niveles de enseñanza, las publicaciones presentes en la revisión y el uso de las palabras claves relacionadas al tema, sin la pretensión de evaluar o disertar sobre todas las estrategias de MA disponibles.

La justificación para identificar cómo se han utilizado las MA en la enseñanza de las Ciencias, basada principalmente en una revisión bibliográfica, se debe al hecho de la conformidad sobre las contribuciones de la investigación bajo el Estado del Conocimiento propuesto por Romanowski y Ens (2006, p. 38), "[...] faltan estudios que equilibren y señalen la necesidad de una revisión que revele y examine el conocimiento elaborado y muestre los enfoques, los temas más investigados y las brechas existentes (traducción propia)".

Entre los estudios de revisión sobre MA, se destacan los trabajos de Nascimento y Coutinho (2016), que revisaron las MA en enseñanza de las Ciencias, además de trabajos específicos sobre MA, como la revisión de la producción académica brasileña sobre el Aula Invertida (VALÉRIO; BELETI JUNIOR, 2019) e la revisión sobre la implementación del Peer Instruction (MÜLLER et al., 2017). Sin embargo, la investigación en transcurso se justifica debido a la necesidad de ampliar la revisión del uso de la MA en la enseñanza de las Ciencias, con el objetivo de señalar los tipos y formas más utilizados, los diferentes enfoques, identificar las posibles tendencias y lagunas, y contribuir para futuros estudios relacionados con el tema. La investigación se configura como un Estado del Conocimiento, como será esclarecido en la sección metodológica.

\section{CONTRIBUCIONES DE LAS MA Y REFLEXIONES SOBRE SU USO}

Las MA favorecen la creación de entornos de aprendizaje en donde los estudiantes tienen un papel destacado durante las clases y actúan de forma más autónoma en la búsqueda de información (MORAN, 2018). Sobre la posición del estudiante como protagonista en la construcción del conocimiento, Valente (2018) argumenta que

La responsabilidad del aprendizaje ahora recae en el estudiante, que necesita
adoptar una postura más participativa, en la que resuelva problemas,
desarrolle proyectos y, con esto, cree oportunidades para desarrollar su
conocimiento. El profesor se convierte en mediador, consultor del aprendiz.
Y el salón de clases se convierte en el lugar donde el estudiante tiene la
presencia del profesor y sus colegas para ayudarlo en la resolución de sus
tareas, el intercambio de ideas y el significado de la información (VALENTE,
2018, p. 42 , traducción propia).

Con este cambio de paradigma, el estudiante se convierte en el centro del proceso de aprendizaje. Se hace necesario estimular su autonomía para que se dé cuenta de que sus ideas y conocimientos previos son válidos en este proceso. 
Cuando el estudiante comprende su contribución, se da cuenta de que es valorado y tiene más libertad de acción durante las clases, el sentimiento de pertenencia puede proporcionar una mayor participación en el contenido que será explicado por el profesor (BERBEL, 2011).

Además, las estrategias desarrolladas con MA amplían las posibilidades de aprendizaje cuando valoran la reflexión, el desarrollo del sentido crítico, la interacción, las discusiones y la participación de los estudiantes, lo que contribuye a despertar el interés en el conocimiento abordado (VALENTE, 2018).

En este sentido, la creación de entornos de discusión, con interacciones entre estudiantes y profesores durante las clases, brinda una mayor oportunidad para los momentos de argumentación de los estudiantes. De acuerdo con Sasseron y Carvalho (2011, p. 100), la argumentación consiste en "toda y cualquiera intervención en que los estudiantes y el profesor presenten sus opiniones en sala de clases, describiendo las ideas, presentando hipótesis y pruebas, que justifiquen las acciones o conclusiones a las que han llegado, explicando los resultados alcanzados (traducción propia)". Para las autoras, la argumentación conduce a la construcción del conocimiento. Por lo tanto, es necesario que el profesor se dé cuenta de la importancia de este proceso y de la creación de entornos que permitan a los estudiantes exponer sus ideas, así como comprender y reconocer su papel como mediador en la conducción de la argumentación durante las clases.

Otro punto importante a considerar en el uso de MA es la influencia y contribución de las tecnologías digitales en los enfoques propuestos por algunas de estas metodologías. Estas tecnologías mejoran los procesos de enseñanza y aprendizaje al facilitar el acceso a la información disponible y a la comunicación, correspondiendo al profesor la mediación entre estos elementos para construir el conocimiento (KENSKI, 2003). Para Cavichia Atanazio y Leite (2018), las tecnologías son herramientas que amplían las oportunidades de aprendizaje, sin embargo, la incorporación y la mediación en el uso de estos instrumentos dependen de la profundización del enfoque por los profesores, de lo contrario, según Silva (2011), puede convertirse en una panacea de métodos y técnicas.

Silva (2011) trae una reflexión sobre el uso descontrolado de los métodos y técnicas, denominado desorden metodológico, disponible y a menudo utilizado sin la planificación de los profesores o sin un enfoque profundo. Para el autor, los profesores usan estrategias para ampliar las posibilidades en el aprendizaje de los estudiantes, sin embargo, en algunos casos, la utilización se encuentra relacionada con el uso de la metodología porque se considera innovadora o implica el uso de tecnologías.

Según Bacich y Moran (2018), es importante pensar en el uso de las MA como un conjunto de posibilidades y no como estrategias, métodos y técnicas utilizadas como secuencias didácticas para enriquecer las clases. Todavía de acuerdo con los autores, las MA no pueden reducirse a estrategias pedagógicas utilizadas como recetas durante las clases. Es necesaria la reflexión y una nueva actitud del profesor que establece una relación con el estudiante, ubicándose como mediador del proceso, dirigiendo a este estudiante a establecer relaciones con sus compañeros y con el objeto de conocimiento.

Teniendo en cuenta estos puntos de vista, nos damos cuenta que existen ventajas relacionadas con el uso de estrategias con MA, sin embargo, los docentes 
y las instituciones educativas deben considerar que los enfoques también pueden presentar limitaciones, siendo importante el (re)conocimiento de los límites en la implementación de MA. Entre las limitaciones discutidas por autores están la dificultad de acceder a las tecnologías digitales en estrategias que dependen de ellas, la falta de preparación de los estudiantes antes de las clases en estrategias como el Aula Invertida (VALENTE, 2014a), y la falta de acceso a materiales actualizados, como referencias bibliográficas en estrategias que dependen de la consulta de estos materiales durante las clases (SOUZA; DOURADO, 2015). La planificación de las clases y el estímulo de los profesores para la argumentación durante los debates en clase son fundamentales en las estrategias que se desarrollan a partir de la resolución de problemas (FREITAS, 2012; SASSERON, 2015). Por lo tanto, es necesario que los profesores estén capacitados para incentivar a los estudiantes en las clases con MA, sin embargo, dadas las falencias que provienen de la formación docente, no siempre los profesores están preparados para actuar como incentivadores y mediadores, lo que puede dificultar la realización de las estrategias.

\section{METOdOLOGÍA}

El presente trabajo corresponde a la primera parte de la disertación de maestría de la primera autora. Para esta investigación tomamos un enfoque cualitativo, constituyéndose como una investigación bibliográfica de revisión bajo el alcance del Estado del Conocimiento. Según Minayo (2009), la investigación cualitativa permite la comprensión e interpretación de la realidad, donde los fenómenos humanos generados socialmente se analizan y explican a partir de datos no cuantificables. Estas investigaciones tienen características que permiten la búsqueda de resultados auténticos y la consideración de las interacciones entre los objetivos basadas en orientaciones teóricas y datos observables. Por lo tanto, la investigación cualitativa tiene aspectos que pueden ayudar a comprender los fenómenos estudiados (SILVEIRA; CORDOVA, 2009).

El trabajo se clasifica como investigación bibliográfica y, en este sentido, según Gil (2002), estas investigaciones se caracterizan por utilizar fuentes ya elaboradas, como libros y artículos científicos. Para el autor, la principal ventaja de la investigación bibliográfica es que permite al investigador un análisis amplio de los fenómenos, especialmente cuando la información que se busca está dispersa en el espacio y el tiempo.

Según Romanovski y Ens (2006), el análisis más detallado en la investigación bibliográfica puede ser en estudios del tipo Estado del Conocimiento. Dichas investigaciones contribuyen al análisis y sistematización de producciones relacionadas con ciertas áreas del conocimiento. Para Ferreira (2002), el Estado del Conocimiento permite la realización de una revisión a partir del análisis y la discusión de la producción académica y científica sobre un tema que, en este caso, son las MA en la enseñanza de las Ciencias. Se puede resaltar la diferencia entre el Estado del Arte, que contempla producciones académicas en un campo específico del conocimiento a partir de estudios como disertaciones, tesis, artículos científicos y publicaciones en eventos; y el Estado del Conocimiento, que contempla sectores específicos de la producción, como los artículos científicos utilizados en este trabajo (FERREIRA, 2002; GAMA RUSSO; RÔÇAS, 2019; 
ROMANOVSKI; ENS, 2006). De esta forma, asumimos esta investigación en el ámbito del Estado del Conocimiento, al tomar las revistas nacionales Qualis A1 del Área de Enseñanza como el sector de las publicaciones a ser analizadas, constituyendo este trabajo en una contribución para la comprensión más amplia sobre las MA en la enseñanza de las Ciencias.

\section{RECOPILACIÓN Y ANÁLISIS DE DATOS}

Inicialmente, para definir las revistas que se consultarán en las búsquedas, se realizó una consulta a la base de datos de Qualis Periodicos disponible a través de la plataforma Sucupira en el portal CAPES, siendo definidos los filtros cuanto a la clasificación de las revistas referentes al cuadrienio 2013-2016 y revistas del Área de Enseñanza. Fueron seleccionadas como base de datos las revistas nacionales, gratuitas y públicas, de nivel $\mathrm{A} 1$, con interés en las revistas que tienen alcance y publicaciones relacionadas o que no excluyan la enseñanza de las Ciencias. En un segundo momento, las páginas de internet de las revistas seleccionadas fueron buscadas a través de Google. En el tercer momento, el tiempo definido para las búsquedas en los volúmenes y números de las publicaciones se delimitó en el período de enero de 2008 a diciembre de 2018. En el cuarto momento: selección de artículos científicos utilizando las palabras claves enumeradas anteriormente y descritas a continuación. La búsqueda se realizó inicialmente en los títulos de los artículos, resúmenes y palabras claves y, luego, a través de la lectura completa del texto para confirmar el uso de MA en la publicación.

La definición de palabras claves se produjo en base a la investigación desarrollada por Berbel $(2011,1998)$, Carvalho (2013), Mazur (2015), Moran (2015, 2018), Queiroz y Cabral (2016) y Valente et al. (2014). Se definió un gran grupo de palabras claves para la selección de artículos, lo que puede justificarse por la ausencia del término metodologías activas en los títulos, palabras claves y/o resúmenes de artículos publicados en revistas. Muchos investigadores, cuando trabajan el tema de MA usan el nombre específico de la metodología investigada en los títulos, palabras claves y resúmenes, por lo tanto, si la búsqueda se reduce al término MA en enseñanza de las Ciencias, varios artículos no serían seleccionados.

Las palabras claves utilizadas en la búsqueda fueron: metodologías activas; metodologías activas y enseñanza de las Ciencias; metodologías activas y Ciencias; Aprendizaje Basado en la Investigación; Enseñanza por Investigación y metodologías activas; actividades de investigación y metodologías activas; Aula Invertida (Flipped classroom); Aula Compartida; Aprendizaje Basado en Problemas (Problem-based learning); Metodología de Problematización y metodologías activas; Metodología de Problematización del arco de Maguerez; Aprendizaje Basado en Proyectos (Project-based learning); Aprendizaje en Equipo (Team-based learning); Aprendizaje Basado en Juegos (Game-based learning); juegos didácticos y metodologías activas; aprendizaje personalizado o personalización; Rotación por Estaciones; Metodología de Contextualización del Aprendizaje; Instrucción por Pares (Peer Instruction); Enseñanza Justo a Tiempo (Just-in-time teaching); Estudios de Casos y metodologías activas; Método de Caso y metodologías activas (Teaching case). 
Asumimos como una forma de análisis inicial de los datos recopilados y revisados la nube de palabras elaborada a partir de las palabras claves descritas en los artículos seleccionados, utilizando el programa disponible en https://www.jasondavies.com/wordcloud/. Las nubes de palabras amplían la visión de ciertos escenarios y permiten identificar palabras más frecuentes en un texto. Por lo tanto, la elaboración de la nube de palabras se dio con el objetivo de visualizar los términos que aparecen con mayor frecuencia en las palabras claves de los artículos.

Para la construcción de la nube de palabras utilizamos términos únicos para representar metodologías activas informadas por los autores con diferentes nomenclaturas. Los términos actividades de investigación, actividad investigativa, actividades investigativas, Aprendizaje Basado en la Investigación y Enseñanza por Investigación han sido reemplazados por la abreviatura E.Inv. (Enseñanza por Investigación). Los términos ABP, resolución de problemas, Aprendizaje Basado en Resolución de Problemas, Problem-based learning y Aprendizaje a partir de Problemas han sido reemplazados por Ensino por Problemas (Enseñanza por Problemas). Los términos Estudios de casos, Aprendizaje Basada en Casos y Aprendizaje Basado en Caso han sido reemplazados por Estudo de Casos. Y finalmente, el término innovaciones pedagógicas ha sido reemplazado por inovação pedagógica.

\section{RESULTADOS Y DISCUSIÓN}

Al considerar el Qualis A1 en el Área de Enseñanza se enumeraron 145 revistas nacionales e internacionales, incluyendo las revistas con duplicidad o triplicidad de título teniendo en cuenta el código de identificación física y digital (International Standard Serial Number - ISSN). Después de la eliminación de revistas internacionales, quedaron 70 revistas nacionales, siendo siete con nombres únicos y 23 con ISSN duplicado o triplicado, y después de la eliminación de repeticiones, quedaron 30 revistas brasileñas. Como el objetivo era la revisión de publicaciones relacionadas con la enseñanza de las Ciencias, se verificó el perfil de cada una de las 30 revistas y no fueron seleccionadas las revistas de las áreas específicas de Salud (04), Matemática (01), Música (01), Historiografía de la Educación (01), Evaluación de la educación (01), Psicología (01), Deporte (01) y Gestión Ambiental (01). En la Tabla 1, presentamos las 19 revistas seleccionadas.

Tabla 1 - Número de publicaciones con las palabras claves en revistas A1 definidas para este estudio desde su alcance

\begin{tabular}{|c|c|c|}
\hline Revistas & $\begin{array}{c}\text { Artículos } \\
\text { investigados }\end{array}$ & $\begin{array}{c}\text { Artículos } \\
\text { seleccionados }\end{array}$ \\
\hline Ensaio: Pesquisa em Educação Em Ciências & 340 & 10 \\
\hline Revista Brasileira de Ensino de Física & 964 & 10 \\
\hline Ciência \& Educação & 603 & 6 \\
\hline Educação e Pesquisa & 656 & 3 \\
\hline Educação e Realidade & 610 & 2 \\
\hline Cadernos de Pesquisa & 490 & 1 \\
\hline Calidoscópio & 339 & 1 \\
\hline
\end{tabular}




\begin{tabular}{|c|c|c|}
\hline Revistas & $\begin{array}{c}\text { Artículos } \\
\text { investigados }\end{array}$ & $\begin{array}{c}\text { Artículos } \\
\text { seleccionados }\end{array}$ \\
\hline Educação em Revista (Unesp-Marília) & 173 & 1 \\
\hline Educação em Revista (UFMG) & 570 & 1 \\
\hline Educar em Revista & 784 & 1 \\
\hline Ensaio - Avaliação e Políticas Públicas em Educação & 430 & 1 \\
\hline Laplage em Revista & 178 & 1 \\
\hline Revista Brasileira de Educação Especial & 417 & 1 \\
\hline Cadernos Cedes & 226 & 0 \\
\hline Currículo sem Fronteiras & 410 & 0 \\
\hline Educação \& Sociedade & 594 & 0 \\
\hline Pro-posições & 405 & 0 \\
\hline Revista Brasileira de Educação & 503 & 0 \\
\hline Total de artículos & 389 & 0 \\
\hline
\end{tabular}

Fuente: Autoría propia (2019).

En la Tabla 2 presentamos las 39 publicaciones relacionadas con las MA y seleccionadas de las palabras claves enumeradas anteriormente. Observamos que las revistas que más presentaron artículos sobre el tema aún no tienen expresión en su producción académica (10 cada una). Una posible justificación para esto es el hecho de que la investigación ha sido limitada en las revistas Qualis A1. Deducimos que la extensión de los límites para las revistas Qualis B1 puede presentar un número significativo de artículos que involucran las MA, ya que dentro de sus objetivos tienden a abarcar informes sobre experiencias. Por otro lado, aunque muchas revistas Qualis A1 no presentan espacio para los informes sobre experiencias, se observa, a través de esta investigación, la falta de artículos que abordan el tema de manera documental y bibliográfica. Entre las publicaciones seleccionadas, se identificó una revisión bibliográfica (MÜLLER et al., 2017).

Tabla 2 - Números de publicaciones con las palabras claves en las revistas A1 definidas para este estudio a partir de sus objetivos

\begin{tabular}{|c|c|c|c|}
\hline \multirow{2}{*}{ Revista - Lugar } & $\begin{array}{c}\text { Volumen, } \\
\text { número e } \\
\text { páginas }\end{array}$ & Título de los artículos & Referencias \\
\hline & $\begin{array}{c}\text { V.13, } \\
\text { n.03, p. } \\
67-80\end{array}$ & $\begin{array}{c}\text { Atividades investigativas no ensino } \\
\text { de Ciências: aspectos históricos e } \\
\text { diferentes abordagens. }\end{array}$ & $\begin{array}{c}\text { ZOMPERO; } \\
\text { LABURÚ, 2011 }\end{array}$ \\
\cline { 2 - 5 } $\begin{array}{c}\text { Ensaio: } \\
\text { Pesquisa em }\end{array}$ & $\begin{array}{c}\text { V.17, n. 3, } \\
\text { E. 555- } \\
\text { Ciências - Belo } \\
\text { Horizonte }\end{array}$ & $\begin{array}{c}\text { Promovendo a alfabetização } \\
\text { científica por meio de ensino } \\
\text { investigativo no ensino médio de } \\
\text { química: contribuições para a } \\
\text { formação inicial docente. }\end{array}$ & $\begin{array}{c}\text { MIRANDA; } \\
\text { SUAR; } \\
\text { MARCONDES, } \\
\text { 2015 }\end{array}$ \\
\cline { 2 - 5 } & $\begin{array}{c}\text { V.17, } \\
\text { n.esp., p. } \\
49-67\end{array}$ & $\begin{array}{c}\text { Alfabetização científica, ensino por } \\
\text { investigação e argumentação: } \\
\text { relações entre ciências da } \\
\text { natureza e escola. }\end{array}$ & SASSERON, 2015 \\
\hline
\end{tabular}




\begin{tabular}{|c|c|c|c|}
\hline & $\begin{array}{c}\text { V.17, } \\
\text { n.esp., p. } \\
97-114\end{array}$ & $\begin{array}{c}\text { Ensino por investigação: eixos } \\
\text { organizadores para sequências de } \\
\text { ensino de biologia. }\end{array}$ & $\begin{array}{l}\text { TRIVELATO; } \\
\text { TONIDANDEL, } \\
2015\end{array}$ \\
\hline \multirow{6}{*}{$\begin{array}{c}\text { Ensaio: } \\
\text { Pesquisa em } \\
\text { Educação Em } \\
\text { Ciências - Belo } \\
\text { Horizonte } \\
\text { (Continuación) }\end{array}$} & $\begin{array}{c}\text { V.17, } \\
\text { n.esp., p. } \\
\text { 115-137 }\end{array}$ & $\begin{array}{l}\text { Sequências didáticas investigativas } \\
\text { e argumentação no ensino de } \\
\text { ecologia. }\end{array}$ & $\begin{array}{l}\text { MOTOKANE, } \\
2015\end{array}$ \\
\hline & $\begin{array}{c}\text { V.18, n. 1, } \\
\text { p. } 123- \\
146\end{array}$ & $\begin{array}{l}\text { Ensino de ciências por } \\
\text { investigação: uma estratégia } \\
\text { pedagógica para promoção da } \\
\text { alfabetização científica nos } \\
\text { primeiros anos do ensino } \\
\text { fundamental. }\end{array}$ & $\begin{array}{c}\text { BRITO; } \\
\text { FIREMAN, } 2016\end{array}$ \\
\hline & $\begin{array}{l}\text { V.18, n.2, } \\
\text { p.73-97 }\end{array}$ & $\begin{array}{l}\text { Ensino orientado para a } \\
\text { aprendizagem baseada na } \\
\text { resolução de problemas e ensino } \\
\text { tradicional: um estudo centrado } \\
\text { em "transformação de matéria e } \\
\text { de energia". }\end{array}$ & $\begin{array}{l}\text { MORGADO; } \\
\text { LEITE; } \\
\text { DOURADO; } \\
\text { FERNANDES; } \\
\text { SILVA, 2016 }\end{array}$ \\
\hline & $\begin{array}{l}\text { V.19, s.n., } \\
\text { e2658 }\end{array}$ & $\begin{array}{c}\text { Espaço interativo de } \\
\text { argumentação colaborativa: } \\
\text { condições criadas pelo professor } \\
\text { para promover argumentação em } \\
\text { aulas investigativas. }\end{array}$ & $\begin{array}{c}\text { FERRAZ; } \\
\text { SASSERON, } 2017\end{array}$ \\
\hline & $\begin{array}{l}\text { V.19, s.n., } \\
\text { e2797 }\end{array}$ & $\begin{array}{l}\text { Jogos tipo "bean bag" em aulas de } \\
\text { evolução. }\end{array}$ & $\begin{array}{c}\text { FERREIRA; SILVA, } \\
2017\end{array}$ \\
\hline & $\begin{array}{l}\text { V.20, s.n., } \\
\text { e9666 }\end{array}$ & $\begin{array}{l}\text { O processo de reflexão orientada } \\
\text { na formação inicial de um } \\
\text { licenciando de química visando o } \\
\text { ensino por investigação e a } \\
\text { promoção da alfabetização } \\
\text { científica. }\end{array}$ & $\begin{array}{c}\text { SUART; } \\
\text { MARCONDES, } \\
2018\end{array}$ \\
\hline \multirow{4}{*}{$\begin{array}{l}\text { Revista } \\
\text { Brasileira de } \\
\text { Ensino de Física } \\
\text { - São Paulo }\end{array}$} & $\begin{array}{l}\text { V. } 32, \mathrm{n} . \\
2,2401\end{array}$ & $\begin{array}{l}\text { Efectos sobre la capacidad de } \\
\text { resolución de problemas de “lápiz } \\
\text { y papel" de una enseñanza- } \\
\text { aprendizaje de la física con una } \\
\text { estructura problematizada. }\end{array}$ & $\begin{array}{l}\text { BECERRA-LABRA; } \\
\text { GRAS-MARTí; } \\
\text { MARTÍNEZ- } \\
\text { TORREGROSA, } \\
2010\end{array}$ \\
\hline & $\begin{array}{l}\text { V. } 34, \mathrm{n} . \\
3,3401\end{array}$ & $\begin{array}{l}\text { Muitos alunos do ensino médio } \\
\text { não querem estudar } \\
\text { física: experiências de } \\
\text { aprendizagem ativa podem mudar } \\
\text { esta atitude negativa! }\end{array}$ & $\begin{array}{l}\text { MARUŠIĆ; } \\
\text { SLIŠKO, } 2012\end{array}$ \\
\hline & $\begin{array}{l}\text { V. } 37, \mathrm{n} . \\
3,3506-1\end{array}$ & $\begin{array}{l}\text { Uma metodologia de } \\
\text { aprendizagem ativa para o ensino } \\
\text { de mecânica em educação de } \\
\text { jovens e adultos. }\end{array}$ & $\begin{array}{l}\text { SANTOS; SASAKI, } \\
2015\end{array}$ \\
\hline & $\begin{array}{l}\text { V. } 39, \mathrm{n} . \\
2, \mathrm{e} 2401\end{array}$ & $\begin{array}{c}\text { Uma associação do método Peer } \\
\text { Instruction com circuitos elétricos } \\
\text { em contextos de aprendizagem } \\
\text { ativa. }\end{array}$ & $\begin{array}{c}\text { ARAUJO; SILVA; } \\
\text { JESUS; OLIVEIRA, } \\
2017\end{array}$ \\
\hline
\end{tabular}




\begin{tabular}{|c|c|c|c|}
\hline \multirow{6}{*}{$\begin{array}{c}\text { Revista } \\
\text { Brasileira de } \\
\text { Ensino de Física } \\
\text { - São Paulo } \\
\text { (Continuación) }\end{array}$} & $\begin{array}{l}\text { V. } 39, \mathrm{n} . \\
2, \mathrm{e} 2403\end{array}$ & $\begin{array}{l}\text { Avaliação de uma metodologia de } \\
\text { aprendizagem ativa em óptica } \\
\text { geométrica através da } \\
\text { investigação das reações dos } \\
\text { alunos. }\end{array}$ & $\begin{array}{c}\text { SASAKI; JESUS, } \\
2017\end{array}$ \\
\hline & $\begin{array}{l}\text { V. } 39, \text { n.3, } \\
\text { e3403 }\end{array}$ & $\begin{array}{l}\text { Uma revisão da literatura acerca } \\
\text { da implementação da metodologia } \\
\text { interativa de ensino Peer } \\
\text { Instruction (1991 a 2015). }\end{array}$ & $\begin{array}{l}\text { MÜLLER; } \\
\text { ARAUJO; VEIT; } \\
\text { SCHELL, } 2017\end{array}$ \\
\hline & $\begin{array}{l}\text { V.39, n.4, } \\
\text { e4405 }\end{array}$ & $\begin{array}{l}\text { Implementação de um aplicativo } \\
\text { para smartphones como sistema } \\
\text { de votação em aulas de Física } \\
\text { com Peer Instruction. }\end{array}$ & $\begin{array}{c}\text { KIELT; SILVA; } \\
\text { MIQUELIN, } 2017\end{array}$ \\
\hline & $\begin{array}{l}\text { V. } 39, \mathrm{n} .4, \\
\text { e4309 }\end{array}$ & $\begin{array}{l}\text { A problem regarding buoyancy of } \\
\text { simple figures suitable for } \\
\text { Problem-Based Learning. }\end{array}$ & $\begin{array}{c}\text { SANTANDER, } \\
2017\end{array}$ \\
\hline & $\begin{array}{l}\text { V. } 40, \mathrm{n} . \\
1, \mathrm{e} 1401\end{array}$ & $\begin{array}{l}\text { Aplicação e avaliação de uma } \\
\text { metodologia de aprendizagem } \\
\text { ativa (tipo ISLE) em aulas de } \\
\text { Mecânica, em cursos de } \\
\text { Engenharia. }\end{array}$ & PARREIRA, 2018 \\
\hline & $\begin{array}{l}\text { V. } 40, n . \\
4, \text { e4403 }\end{array}$ & $\begin{array}{l}\text { Atividades investigativas e o } \\
\text { desenvolvimento de habilidades e } \\
\text { competências: um relato de } \\
\text { experiência no curso de Física da } \\
\text { Universidade Federal do Pará. }\end{array}$ & $\begin{array}{l}\text { FRAIHA; } \\
\text { PASCHOAL JR; } \\
\text { PEREZ; TABOSA; } \\
\text { ALVES; SILVA, } \\
2018\end{array}$ \\
\hline \multirow{6}{*}{$\begin{array}{l}\text { Ciência \& } \\
\text { Educação - } \\
\text { Bauru }\end{array}$} & $\begin{array}{l}\text { V. } 14, \mathrm{n} . \\
\text { 3, p. } 555- \\
74\end{array}$ & $\begin{array}{c}\text { Estudos de caso como estratégia } \\
\text { de ensino na formação de } \\
\text { professores de física. }\end{array}$ & $\begin{array}{c}\text { LINHARES; REIS, } \\
2008\end{array}$ \\
\hline & $\begin{array}{l}\text { V. } 16, \mathrm{n} . \\
1, \mathrm{p} .181- \\
198\end{array}$ & $\begin{array}{l}\text { O uso de metodologias ativas no } \\
\text { ensino de graduação nas ciências } \\
\text { sociais e da saúde: avaliação dos } \\
\text { estudantes. }\end{array}$ & $\begin{array}{c}\text { GOMES; } \\
\text { RIBEIRO; } \\
\text { MONTEIRO; } \\
\text { LEHER; } \\
\text { LOUZADA, } 2010 \\
\end{array}$ \\
\hline & $\begin{array}{l}\text { V. } 19, \mathrm{n} . \\
\text { 3, p. } 499- \\
516\end{array}$ & $\begin{array}{c}\text { EI ABP y el diagrama heurístico } \\
\text { como herramientas para } \\
\text { desarrollar la argumentación } \\
\text { escolar en las asignaturas de } \\
\text { ciencias. }\end{array}$ & $\begin{array}{l}\text { CAMPILLO; } \\
\text { GUERRERO; } \\
2013\end{array}$ \\
\hline & $\begin{array}{l}\text { V. } 22, \mathrm{n} . \\
2, \text { p. } 319- \\
333\end{array}$ & $\begin{array}{l}\text { A percepção de professores e } \\
\text { alunos do ensino médio sobre a } \\
\text { atividade estudo de caso. }\end{array}$ & $\begin{array}{c}\text { FARIA; FREITAS- } \\
\text { REIS, } 2016\end{array}$ \\
\hline & $\begin{array}{l}\text { V. } 23, \mathrm{n} . \\
2, \text { p. } 419- \\
\quad 436\end{array}$ & $\begin{array}{l}\text { Atividades de investigação na } \\
\text { disciplina de Ciências e } \\
\text { desenvolvimento de habilidades } \\
\text { cognitivas relacionadas a funções } \\
\text { executivas. }\end{array}$ & $\begin{array}{l}\text { ZOMPERO; } \\
\text { GONÇALVES; } \\
\text { LABURÚ, } 2017\end{array}$ \\
\hline & $\begin{array}{l}\text { V. } 23, \mathrm{n} . \\
\text { 3, p. } 659- \\
\quad 676\end{array}$ & $\begin{array}{l}\text { Atividades de investigação e a } \\
\text { transferência de significados sobre } \\
\text { o tema educação alimentar no } \\
\text { ensino fundamental. }\end{array}$ & $\begin{array}{l}\text { ZOMPERO; } \\
\text { FIGUEIREDO; } \\
\text { GARBIM, } 2017\end{array}$ \\
\hline $\begin{array}{c}\text { Educação e } \\
\text { Pesquisa - São } \\
\text { Paulo }\end{array}$ & $\begin{array}{l}\text { V. } 38, \text { n.2, } \\
\text { p. } 403- \\
418\end{array}$ & $\begin{array}{l}\text { Ensino por problemas: uma } \\
\text { abordagem para o } \\
\text { desenvolvimento do aluno. }\end{array}$ & FREITAS, 2012 \\
\hline
\end{tabular}




\begin{tabular}{|c|c|c|c|}
\hline & $\begin{array}{l}\text { V. } 40, \text { n. } \\
\text { 3, p. } 637- \\
\quad 650\end{array}$ & $\begin{array}{l}\text { Proposta didática para o curso de } \\
\text { licenciatura em educação física: } \\
\text { aprendizagem baseada em casos. }\end{array}$ & METZNER, 2014 \\
\hline & $\begin{array}{l}\text { V. } 41, \mathrm{n} . \\
\text { 3, p. } 713- \\
728\end{array}$ & $\begin{array}{l}\text { A aprendizagem contextualizada: } \\
\text { análise dos seus fundamentos e } \\
\text { práticas pedagógicas. }\end{array}$ & FESTAS, 2015 \\
\hline \multirow{2}{*}{$\begin{array}{l}\text { Educação e } \\
\text { Realidade - } \\
\text { Porto Alegre }\end{array}$} & $\begin{array}{l}\text { V. } 35, \text { n.1, } \\
\text { p.129-150 }\end{array}$ & $\begin{array}{l}\text { Ensino de Ciências com } \\
\text { Tecnologias: um caminho } \\
\text { metodológico no PROEJA. }\end{array}$ & $\begin{array}{l}\text { REIS; LINHARES, } \\
2010\end{array}$ \\
\hline & $\begin{array}{l}\text { V.43, n.2, } \\
\text { p.551- } \\
568\end{array}$ & $\begin{array}{c}\text { Estetização Pedagógica, } \\
\text { Aprendizagens Ativas e Práticas } \\
\text { Curriculares no Brasil. }\end{array}$ & SILVA, 2018 \\
\hline $\begin{array}{l}\text { Cadernos de } \\
\text { Pesquisa - São } \\
\text { Paulo }\end{array}$ & $\begin{array}{c}\text { V.45, } \\
\text { n.156, } \\
\text { p.376-389 }\end{array}$ & $\begin{array}{l}\text { Aprendizaje basado en problemas } \\
\text { en la formación de psicólogos. }\end{array}$ & $\begin{array}{l}\text { HERRERA; } \\
\text { OPAZO, } 2015\end{array}$ \\
\hline $\begin{array}{l}\text { Calidoscópio - } \\
\text { Rio Grande do } \\
\quad \text { Sul }\end{array}$ & $\begin{array}{l}\text { V. } 14, \mathrm{n} . \\
3, \text { p. } 413- \\
\quad 422\end{array}$ & $\begin{array}{l}\text { Objetos digitais para a } \\
\text { aprendizagem da leitura: uma } \\
\text { metodologia ativa de ensino. }\end{array}$ & $\begin{array}{c}\text { MARTINS; } \\
\text { FORNECK; } \\
\text { DIESEL; BUBLITZ, } \\
2016\end{array}$ \\
\hline $\begin{array}{l}\text { Educação em } \\
\text { Revista (UNESP) } \\
\text { - Marília }\end{array}$ & $\begin{array}{l}\text { V.17, } \\
\text { ed.esp., p. } \\
\text { 39-52 }\end{array}$ & $\begin{array}{l}\text { Formação continuada e o uso de } \\
\text { metodologias ativas de } \\
\text { aprendizagem: as contribuições do } \\
\text { Pacto Nacional pela Alfabetização } \\
\text { na Idade Certa para a atuação de } \\
\text { orientadores de estudo. }\end{array}$ & $\begin{array}{l}\text { KLEIN; GALINDO; } \\
\text { FIDELIS; PASSOS, } \\
2016\end{array}$ \\
\hline $\begin{array}{c}\text { Educação em } \\
\text { Revista (UFMG) } \\
\text { - Belo } \\
\text { Horizonte }\end{array}$ & $\begin{array}{l}\text { V.27, } \\
\text { n.03, } \\
\text { p.61-78 }\end{array}$ & $\begin{array}{l}\text { Metodologia Problematizadora e } \\
\text { suas implicações para a atuação } \\
\text { docente: relato de experiência. }\end{array}$ & $\begin{array}{l}\text { CORRÊA; } \\
\text { SANTOS; SOUZA; } \\
\text { CLAPIS, } 2011\end{array}$ \\
\hline $\begin{array}{c}\text { Educar em } \\
\text { Revista - } \\
\text { Curitiba }\end{array}$ & $\begin{array}{l}\text { S/v., ed. } \\
\text { Esp., n. 4, } \\
\text { p. 79-97 }\end{array}$ & $\begin{array}{c}\text { Blended learning e as mudanças } \\
\text { no ensino superior: a proposta da } \\
\text { sala de aula invertida. }\end{array}$ & VALENTE, 2014 a \\
\hline $\begin{array}{c}\text { Ensaio: } \\
\text { Avaliação e } \\
\text { Políticas } \\
\text { públicas em } \\
\text { Educação - Rio } \\
\text { de Janeiro }\end{array}$ & $\begin{array}{l}\text { V.22, n. } \\
83, \text { p. } \\
263-294\end{array}$ & $\begin{array}{l}\text { Aprendizagem Baseada em } \\
\text { Problemas: um método de ensino- } \\
\text { aprendizagem e suas práticas } \\
\text { educativas. }\end{array}$ & $\begin{array}{c}\text { BOROCHOVICIUS } \\
\text {; TORTELLA, } \\
2014\end{array}$ \\
\hline $\begin{array}{l}\text { Laplage em } \\
\text { Revista - } \\
\text { Sorocaba }\end{array}$ & $\begin{array}{l}\text { V.4, n.3, } \\
\text { p.180-189 }\end{array}$ & $\begin{array}{l}\text { Metodologias de ensino para a } \\
\text { formação de engenheiros no } \\
\text { ensino superior: uma revisão } \\
\text { sistemática. }\end{array}$ & $\begin{array}{l}\text { PEREIRA; DOS } \\
\text { SANTOS JUNIOR, } \\
2018\end{array}$ \\
\hline $\begin{array}{l}\text { Revista } \\
\text { Brasileira de } \\
\text { Educação } \\
\text { Especial - } \\
\text { Marília }\end{array}$ & $\begin{array}{l}\text { V. } 21, \mathrm{n} . \\
1, \text { p. } 143- \\
158\end{array}$ & $\begin{array}{c}\text { Aprendizaje Basado en Juegos } \\
\text { Digitales en Niños con TDAH: un } \\
\text { Estudio de Caso en la Enseñanza } \\
\text { de Estadística para Estudiantes de } \\
\text { Cuarto Grado en Colombia. }\end{array}$ & $\begin{array}{l}\text { MORENO; } \\
\text { VALDERRAMA, } \\
2015\end{array}$ \\
\hline
\end{tabular}

Fuente: Autoría propia (2019).

Presentamos en la Tabla 3 las MA mencionadas en los artículos, algunas de las cuales fueron seleccionados en las búsquedas a través de la palabra clave metodologías activas y no por el nombre específico. Entre estos encontramos Predecir-Observar-Explicar (POE); Objeto Digital Educativo (ODE), Pensaremparejar-compartir (Think-Pair-Share); Reading, presenting and questioning 
(RPQ) y Experimenting and Discussion (ED); Teaching-based on research y Investigative Science Learning Environment (ISLE). Otras MA previamente enumeradas en el grupo de palabras claves por sus nombres específicos, pero no encontradas incluyen: Aula Compartida, Aprendizaje Basado en Proyectos y en Equipo, Aprendizaje Personalizado, Rotación por Estaciones y Enseñanza Justo a Tiempo. En la Tabla 3, las MA más destacadas son Enseñanza por Problemas (ABP y MP) y Enseñanza por Investigación. Fue encontrada una mayor frecuencia de estas estrategias, en principio, porque inferimos que están asociadas con los procesos de argumentación y Alfabetización Científica. Según Trivelato y Tonidandel (2015), la argumentación fomenta el aprendizaje de conceptos científicos y aumenta la participación de los estudiantes. Para Motokane (2015), La Enseñanza por Investigación fomenta la argumentación, siendo este proceso fundamental para la comprensión de cómo el estudiante se apropia del conocimiento científico, contribuyendo al proceso de Alfabetización Científica, en el que el estudiante comienza a percibir el mundo desde la perspectiva de la Ciencia.

Tabla 3 - Metodologías activas en artículos seleccionados

\begin{tabular}{|c|c|c|}
\hline Metodologías activas & $\begin{array}{l}\text { Número de } \\
\text { artículos }\end{array}$ & Referencias \\
\hline Enseñanza por Investigación & 11 & $\begin{array}{l}\text { BRITO; FIREMAN, 2016; FERRAZ; } \\
\text { SASSERON, 2017; FRAIHA et al., 2018; } \\
\text { MIRANDA et al., 2015; MOTOKANE, } \\
\text { 2015; SASSERON, 2015; SUART; } \\
\text { MARCONDES, 2018; TRIVELATO; } \\
\text { TONIDANDEL, 2015; ZOMPERO; } \\
\text { FIGUEIREDO; GARBIM, 2017; } \\
\text { ZOMPERO; GONÇALVES; LABURÚ, } \\
\text { 2017; ZOMPERO; LABURÚ, } 2011\end{array}$ \\
\hline $\begin{array}{l}\text { Enseñanza por Problemas: } \\
\text { Aprendizaje Basada en } \\
\text { Problemas; } \\
\text { Metodología de la } \\
\text { Problematización }\end{array}$ & 10 & $\begin{array}{l}\text { BECERRA-LABRA et al., 2010; } \\
\text { BOROCHOVICIUS; TORTELLA, 2014; } \\
\text { CAMPILLO; GUERRERO, 2013; CORRÊA } \\
\text { et al., 2011; FREITAS, 2012; GOMES et } \\
\text { al., 2010; HERRERA; OPAZO, 2015; } \\
\text { KLEIN et al. 2016; MORGADO et al., } \\
\text { 2016; SANTANDER, } 2017\end{array}$ \\
\hline Estudio de Casos & 04 & $\begin{array}{c}\text { FARIA; FREITAS-REIS, 2016; LINHARES; } \\
\text { REIS, 2008; METZNER, 2014; REIS; } \\
\text { LINHARES, 2010; }\end{array}$ \\
\hline Instrucción por Pares & 03 & $\begin{array}{c}\text { ARAUJO et al., 2017; KIELT et al., 2017, } \\
\text { MÜLLER et al., } 2017\end{array}$ \\
\hline $\begin{array}{l}\text { Predecir-Observar-Explicar } \\
\text { (POE) }\end{array}$ & 02 & $\begin{array}{c}\text { SANTOS; SASAKI, 2015; SASAKI; JESUS, } \\
2017\end{array}$ \\
\hline $\begin{array}{c}\text { Aprendizaje Basada en } \\
\text { Juegos }\end{array}$ & 02 & $\begin{array}{c}\text { FERREIRA; SILVA, 2017; MORENO; } \\
\text { VALDERRAMA, } 2015\end{array}$ \\
\hline $\begin{array}{c}\text { Instrucción por Pares; } \\
\text { Pensar-emparejar-compartir } \\
\text { (Think-Pair-Share); } \\
\text { Aprendizaje Basado en } \\
\text { Equipos }\end{array}$ & 01 & SILVA, 2018 \\
\hline
\end{tabular}




\begin{tabular}{|c|c|c|}
\hline Metodologías activas & $\begin{array}{l}\text { Número de } \\
\text { artículos }\end{array}$ & Referencias \\
\hline Aula Invertida & 01 & VALENTE, 2014 a \\
\hline Aprendizaje Contextualizado & 01 & FESTAS, 2015 \\
\hline Teaching-based on research & 01 & PEREIRA; DOS SANTOS JUNIOR, 2018 \\
\hline $\begin{array}{l}\text { Reading, presenting and } \\
\text { questioning (RPQ) y } \\
\text { Experimenting and Discussion } \\
\text { (ED) }\end{array}$ & 01 & MARUŠIĆ; SLIŠKO, 2012 \\
\hline $\begin{array}{c}\text { Investigative Science Learning } \\
\text { Environment (ISLE) }\end{array}$ & 01 & PARREIRA, 2018 \\
\hline Objeto Digital Educativo & 01 & MARTINS et al., 2016 \\
\hline
\end{tabular}

Fuente: Autoría propia (2019).

Los niveles de educación más utilizados por los autores para la aplicación de las MA comprenden 14 artículos en Educación Superior (BECERRA-LABRA et al., 2010; BOROCHOVICIUS; TORTELLA, 2014; CAMPILLO; GUERRERO, 2013; CORRÊA et al., 2011; FERREIRA; SILVA, 2017; FRAIHA et al., 2018; GOMES et al., 2010; HERRERA; OPAZO, 2015; LINHARES; REIS, 2008; METZNER, 2014; PARREIRA, 2018; PEREIRA; DOS SANTOS JUNIOR, 2018; SANTANDER, 2017; VALENTE, 2014a) y 11 artículos en la Escuela Secundaria (ARAUJO et al., 2017; FARIA; FREITAS-REIS, 2016; KIELT et al., 2017; MARUŠIĆ; SLIŠKO, 2012; MIRANDA et al., 2015; REIS; LINHARES, 2010; SANTOS; SASAKI, 2015; SASAKI; JESUS, 2017; SILVA, 2018; SUART; MARCONDES, 2018; ZOMPERO; GONÇALVES; LABURÚ, 2017).

El mayor número de publicaciones en Educación Superior puede explicarse, según Müller et al. (2017), por la presencia de grupos de investigación en instituciones de nivel Superior, lo que favorece el desarrollo de trabajos en este entorno. Además, según los autores, la Educación Superior y la Educación Secundaria enfrentan varias dificultades en el aprendizaje y la motivación de los estudiantes, lo que favorece el desarrollo de la investigación en busca de nuevas metodologías de enseñanza y sus ventajas para el aprendizaje. Por otro lado, hay una falta de investigación en la Escuela Primaria sobre las MA en la enseñanza de las Ciencias. En este estudio, se identificaron seis artículos relacionados con este nivel de educación (BRITO; FIREMAN, 2016; FERRAZ; SASSERON, 2017; MARTINS et al., 2016; MORENO; VALDERRAMA, 2015; MORGADO et al., 2016; ZOMPERO; FIGUEIREDO; GARBIM, 2017). Una posible justificación para este hecho puede ser el límite realizado en esta investigación, ya que se puede haber eliminado la posibilidad de que surjan reflexiones y experiencias de las MA en la Escuela Primaria. Otra posible justificación puede estar relacionada con la presencia de investigadores en Educación Superior, lo que favorece el desarrollo de investigaciones que no aborden el nivel Primario, como se mencionó anteriormente.

Observando los números de publicaciones en la revisión de 2008 a 2018, fue visto que en los años de 2015 a 2018, de los periódicos con mayor número de publicaciones relacionadas a las MA la Revista Brasileira de Ensino de Física y la Revista Ensaio: Pesquisa em Educação em Ciências, ambas con un total de 17 artículos, entre los 27 publicados de 2015 a 2018. El objetivo de la Revista Ensaio: Pesquisa em Educação em Ciências incluye el interés centrado en la publicación de 
informes de investigación, revisión crítica de la literatura y debates sobre temas relacionados con la educación en Ciencias Naturales en todos los niveles de enseñanza. Por lo tanto, las nueve publicaciones destacadas pueden relacionarse con el objetivo de la revista y al año 2015, donde se publicaron tres artículos en la edición temática sobre argumentación y Enseñanza de las Ciencias. La Revista Brasileira de Ensino de Física tiene ocho publicaciones entre 2015 y 2018, resaltando el año de 2017 con cinco artículos, cuya meta incluye objetivos destinados a una audiencia de investigadores, estudiantes de postgrado, profesores de Física en general y a la comunidad que opera en la investigación y desarrollo de metodologías y materiales para la enseñanza de la Física. Por lo tanto, el aumento de las publicaciones en 2017 sobre la enseñanza de la Física en la revista puede estar justificado por la mayor cantidad de artículos sobre las MA, como Instrucción por Pares y Predecir-Observar-Explicar (POE), que están relacionados con la enseñanza de la Física y el interés de la revista sobre este tema.

Figura 1 - Nube de palabras elaborada con palabras claves de artículos

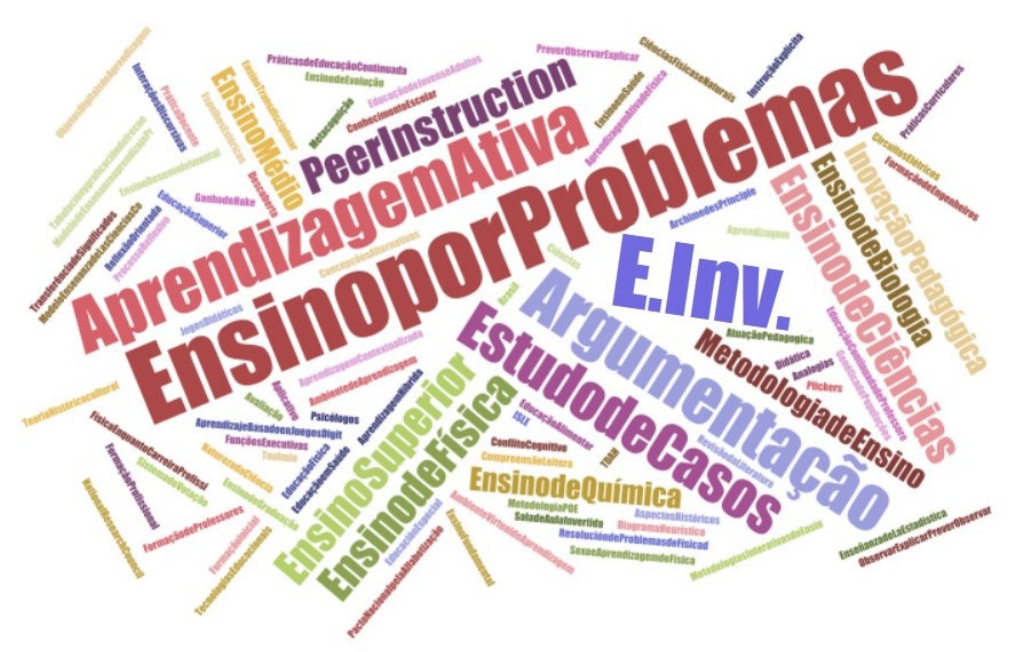

Fuente: Autoría propia (2019).

Presentamos en la Figura 1 la nube de palabras elaborada a partir de las palabras claves descritas en los artículos. Seleccionamos las palabras resaltadas en la nube, con E.Inv. (Enseñanza por Investigación) y Ensino por Problemas (Enseñanza por Problemas) como los términos más destacados. Como ya se discutió, estas metodologías se resaltan porque son estrategias destinadas al desarrollo de las habilidades de resolución de problemas en los estudiantes y están vinculadas a los procesos de argumentación y de Alfabetización Científica. Además de los aspectos más resaltados, el Estudio de Caso también se presenta como una variación de Aprendizaje por Problemas y se incluye en este grupo.

Los términos argumentação (argumentación) y aprendizagem ativa (aprendizaje activo) también son resaltados en la nube, lo que significa que son frecuentes en las palabras claves de los artículos. De acuerdo con Valente et al. (2017), el aprendizaje activo está relacionado con la participación del estudiante en actividades, como protagonistas de su aprendizaje. Por otro lado, el proceso de argumentación puede conducir a la apropiación del conocimiento científico, permitiendo al estudiante percibir los fenómenos a partir de entendimientos científicos (MOTOKANE, 2015). Por lo tanto, los términos representan procesos 
fundamentales en el desarrollo de las MA y el uso de palabras claves en los artículos demuestra que los autores son conscientes de la importancia de la participación e interés de los estudiantes en los procesos de enseñanza y aprendizaje.

El destaque para Instrucción por Pares se da por el vínculo con la enseñanza de la Física, que puede explicarse por el origen de la metodología en esta área del conocimiento (MAZUR, 2015; MÜLLER et al.2017). Kielt et al. (2017) y Araujo et al. (2017) utilizaron la Instrucción por Pares para la enseñanza de conceptos de Física en contextos de aprendizaje activo. La metodología está relacionada con el aprendizaje activo porque es un enfoque que, según Mazur (2015), se basa en la interacción entre pares para resolver preguntas, colocando al estudiante en el centro del proceso de aprendizaje.

La enseñanza de la Física también se distingue por la frecuencia de las MA asociadas con la disciplina en los artículos, como las metodologías de PredecirObservar-Explicar (POE) (SANTOS; SASAKI, 2015; SASAKI; JESUS, 2017); Investigative Science Learning Environment (ISLE) (PARREIRA, 2018); Reading, Presenting and Questioning (RPQ) y Experimenting and Discussion (ED) (MARUŠIĆ; SLIŠKO, 2012). Algunas de estas MA se originaron en la enseñanza de la Física, como la Instrucción por Pares de Eric Mazur, la ISLE, desarrollada por Eugenia Etkina en 2014 para el aprendizaje activo de conceptos de Física a partir de experimentos, y el POE, creado inicialmente como una propuesta de evaluación, sin embargo, asociado a la enseñanza de la Física en varios estudios (PARREIRA, 2018).

Otros términos resaltados incluyen la enseñanza de las Ciencias, la enseñanza de la Biología y la enseñanza de la Química, lo que demuestra que las MA se han desarrollado en las asignaturas relacionadas con la enseñanza de las Ciencias Naturales. Deducimos que el término innovación pedagógica está relacionado con la introducción de metodologías como nuevas posibilidades para la enseñanza. Además, el mayor número de destacados para la Escuela Secundaria y la Universidad se deben a la frecuencia con que las MA son trabajadas en estos niveles de educación.

\section{CONSIDERACIONES FINALES}

La historia del uso de las MA en la enseñanza no es reciente y muchas instituciones y profesores han desarrollado trabajos con las MA en busca de mejores resultados en el aprendizaje de los estudiantes. Según Moran (2018), existe una variedad de estrategias de MA que se pueden desarrollar en la enseñanza y que amplían las posibilidades de participación, autonomía y participación de los estudiantes en los procesos de enseñanza y aprendizaje. En este sentido enfatizamos que, entre la diversidad de metodologías y enfoques de enseñanza, las estrategias con MA constituyen posibilidades para el aprendizaje activo.

En el análisis buscamos reflexiones sobre la importancia de profundizar en las MA, para evitar su uso como desorden metodológico, porque según Silva (2011), los desafíos en el aprendizaje no pueden resolverse reemplazando métodos más antiguos por métodos considerados nuevos, siguiendo las tendencias. En el trabajo 
con las MA, las instituciones educativas y los profesores deben estar atentos a la planificación de las clases, a los pasos didácticos de los enfoques, al conocimiento del público involucrado y a la actitud del profesor como mediador (LACANALLO et al., 2007; MORAN, 2018). Sin embargo, la preparación del profesor para actuar como mediador no siempre ocurre en la formación docente inicial. Además, existen desafíos relacionados con la formación continuada que dificultan la actuación de los profesores para reflexionar sobre su práctica pedagógica. Por lo tanto, en base a las preguntas abordadas en este trabajo, proponemos reflexiones sobre el uso apropiado de las MA, sin la intención de presentarlas como estrategias milagrosas en la enseñanza y de hacer que solo el profesor sea responsable del uso adecuado, porque en los procesos de enseñanza y aprendizaje hay varios factores a considerar y varios actores involucrados.

Dadas las reflexiones, volvemos a la pregunta que guió el desarrollo de la investigación: ¿Cómo el tema de las MA ha sido utilizado y discutido por profesores e investigadores en el Área de la Enseñanza de las Ciencias? Buscando identificar los contextos de desarrollo de las MA, en las 39 publicaciones relacionadas con las MA y la enseñanza de las Ciencias, los resultados muestran que las estrategias más trabajadas por los autores son Enseñanza por Problemas y Enseñanza por Investigación, y estos enfoques son importantes para fomentar la argumentación y llevar a los estudiantes al aprendizaje activo. También enfatizamos que los niveles de educación más sobresalientes pueden estar relacionados con los lugares de trabajo de los autores y que el aumento en el número de publicaciones de 2015 a 2018 puede estar relacionado con el objetivo de las revistas y las ediciones especiales que involucran el tema. Además, el análisis de las palabras claves utilizadas en los artículos nos permite inferir que los autores que desarrollan las MA son conscientes de la importancia del aprendizaje activo y la argumentación en la enseñanza de las Ciencias.

En este trabajo, algunos artículos fueron escogidos debido a los criterios de selección de las revistas, que no excluyen la enseñanza de las Ciencias (GOMES et al., 2010; METZNER, 2014; HERRERA; OPAZO, 2015; MARTINS et al., 2016; CORRÊA et al., 2011; BOROCHOVICIUS; TORTELLA, 2014; PEREIRA; DOS SANTOS JUNIOR, 2018; MORENO; VALDERRAMA, 2015), y aunque no están directamente relacionados con el área, contribuyen a la formación de profesores y se aproximan del Área de Enseñanza en Ciencias.

Delante de los resultados y reflexiones en el análisis de los datos, concluimos que, en los trabajos seleccionados, los autores han discutido diferentes estrategias de las MA en diferentes niveles de educación y en los propios estudios, los autores se citan en círculo, sin ampliar las discusiones con otros pares. En la búsqueda para comprender cómo se han utilizado las MA en la enseñanza de Ciencias y cómo se produce el flujo de información entre profesores e investigadores, se necesitan estudios más amplios para identificar cómo interactúan los autores con sus pares, cómo se intercambia el conocimiento entre las instituciones de diferentes lugares y regiones y si las discusiones se basan en referencias bibliográficas primarias, es decir, en autores con trabajos consolidados en el área. Considerando el objetivo de este trabajo, proponemos reflexiones sobre el uso de MA en la enseñanza, profundizando en aspectos que responden a la pregunta de investigación y revelan los contextos de enseñanza en los que se desarrollan las estrategias, sin embargo, el tema no puede agotarse en un análisis y se pueden realizar nuevas 
investigaciones teniendo en cuenta la diversidad de sectores de organización de producciones académicas. De acuerdo con los datos obtenidos, inferimos que la expansión de la búsqueda en las revistas Qualis B1 puede traer nuevos entendimientos sobre los contextos de desarrollo de las MA, lo que requiere estudios futuros que cubran las revistas clasificadas con este nuevo límite. Esta apuesta en las revistas B1 se debe al hecho de que los objetivos de estas revistas hacen posible que los profesores compartan sus experiencias y relatos de las clases. De hecho, esto fue atribuido como una de las posibles justificaciones para la baja demanda de publicaciones en las revistas revisadas y también inferimos que faltan artículos que abordan el tema de manera bibliográfica, lo que constituye, una producción académica aún inexpresiva sobre el tema en las revistas Qualis A1 del Área de Enseñanza de las Ciencias. Por lo tanto, a partir de la revisión inicial realizada en este trabajo, tenemos la intención de ampliar el estudio para un análisis más profundo, buscando comprender como fluye la información sobre las MA entre autores e instituciones, a través de redes de conocimiento sobre el tema. 


\title{
Metodologias ativas no ensino de ciências: mapeamento e análise das publicações em revistas da área de ensino na década de 2008 a 2018
}

\begin{abstract}
RESUMO
Este trabalho apresenta um mapeamento de artigos científicos sobre metodologias ativas publicados em revistas nacionais Qualis A1 da Área de Ensino da Coordenação de Aperfeiçoamento de Pessoal de Nível Superior (CAPES). Para responder à pergunta: Como o tema metodologias ativas vem sendo utilizado e discutido pelos professores e pesquisadores no ensino de Ciências?, o trabalho tem por objetivo realizar o levantamento dos artigos relacionados ao ensino de Ciências no período de 2008 a 2018. Os objetivos específicos incluem selecionar publicações e mapear os contextos de ensino em que as estratégias são desenvolvidas, sem a pretensão de avaliar ou discorrer sobre todas as estratégias de metodologias ativas disponíveis. Para isso, foi assumida abordagem qualitativa, sendo a pesquisa bibliográfica realizada no âmbito do Estado do Conhecimento. As revistas nacionais foram selecionadas a partir da consulta ao portal da CAPES, na plataforma Sucupira, utilizando os filtros: Área de Ensino e quadriênio 2013-2016; e para a seleção dos artigos foram definidas palavras-chave relacionadas às metodologias ativas. Como resultados, foram selecionados dezenove revistas e um total de trinta e nove publicações. As metodologias ativas mais frequentes nos artigos são o Ensino por Problemas e o Ensino por Investigação, e os níveis de ensino que mais se destacam são o Ensino Superior e o Ensino Médio. Na análise temporal deste trabalho houve aumento no número de publicações nos anos de 2015 a 2018. Concluímos que os professores e pesquisadores têm discutido as metodologias ativas por meio de diferentes estratégias, em diversos níveis de ensino. Salientamos a importância do aprofundamento teórico para o desenvolvimento de trabalho pautado no uso de metodologias ativas, evitando uma utilização equivocada ou descontrolada, constituindo-se, dessa forma, em uma barafunda metodológica.
\end{abstract}

PALAVRAS-CHAVE: Metodologias ativas. Ensino de Ciências. Estado do Conhecimento. Pesquisa Bibliográfica. 


\title{
Active methodologies in science teaching: mapping and analysis of publications in periodicals in the área de ensino from 2008 to 2018
}

\begin{abstract}
This work presents a review of scientific articles on active methodologies published in Brazilian journals Qualis A1's Área de Ensino, one of the 49 knowledge areas of the Coordenação de Aperfeiçoamento de Pessoal de Nível Superior (CAPES). To answer the question: How has the theme of active methodologies has been used and discussed by teachers and researchers in science education?, the work aims to survey the articles related to science education from 2008 to 2018. Specific objectives include selecting publications and mapping the teaching contexts in which the strategies are developed, without the intention of evaluating or discussing all available active methodologies strategies. For this, it was adopted qualitative approach and the research was carried out under the scope of the State of Knowledge. The national journals were selected by consulting the CAPES portal, on the Sucupira platform, using the filters: Área de Ensino and 2013-2016 quadrennium; keywords related to the active methodologies were defined for the articles selection. As result, were selected nineteen journals and thirty-nine publications. The most frequent active methodologies in the articles are Teaching for Problems and Teaching by Investigation, with a highlighted for education levels of Higher Education and High School. In the period of this work, there was an increase in the number of publications from 2015 to 2018. We conclude that teachers and researchers have discussed active methodologies through different strategies, at different levels of education. We emphasize the importance of theoretical deepening in the use of active methodologies, avoiding misuse or uncontrolled use, such as a methodological disorder.
\end{abstract}

KEYWORDS: Active Methodologies. Science Education. State of Knowledge. Bibliographic Research. 


\section{AGRADECIMIENTOS}

Los autores agradecen a la CAPES por el apoyo financiero que posibilitó esta investigación y agradecen a Javier A. G. Goméz por la revisión en la traducción.

\section{REFERENCIAS}

ARAUJO, I. S.; MAZUR, E. Instrução pelos Colegas e Ensino Sob Medida: uma proposta para o engajamento dos alunos no processo de ensino-aprendizagem de Física. Caderno Brasileiro de Ensino de Física, Florianópolis, v. 30, n. 2: p. 362384, ago. 2013. Disponível em: https://periodicos.ufsc.br/index.php/fisica/article/view/21757941.2013v30n2p362. Acesso em: 20 out. 2019.

BACICH, L.; MORAN, J. (Orgs.). Metodologias ativas para uma educação inovadora: uma abordagem teórico-prática. Porto Alegre, Penso, 2018. 238 p.

BELTRÃO, R. C.; SOUZA, C. M. P.; SILVA, C. P. S. Contrato Didático e suas influências na sala de aula. Educação Matemática Pesquisa, São Paulo, v. 12, n. 2, p.335-353, 2010. Disponível em:

https://revistas. pucsp.br/index.php/emp/article/viewFile/2812/3309. Acesso em: 02 nov. 2019.

BERBEL, N. A. N. As metodologias ativas e a promoção da autonomia de estudantes. Semina: Ciências Sociais e Humanas, Londrina, v. 32, n. 1, p. 25-40, jan./jun. 2011. Disponível em: http://www.uel.br/revistas/uel/index.php/ seminasoc/article/view/10326/0. Acesso em: 20 out. 2019.

BERBEL, N. A. N. A problematização e a aprendizagem baseada em problemas: diferentes termos ou diferentes caminhos? Interface - Comunicação, Saúde, Educação, Botucatu, v. 2, n.2, p. 139-154, 1998. Disponível em: http://www.scielo.br/pdf/icse/v2n2/08.pdf. Acesso em: 20 out. 2019.

BERGMANN, J.; SAMS, A. Sala de aula invertida: uma metodologia ativa de aprendizagem. Rio de Janeiro, LTC, 2016.

CARVALHO, A. M. P. O ensino de Ciências e a proposição de sequências de ensino investigativas. In: CARVALHO, A. M. P. (Org.). Ensino de Ciências por Investigação: condições para implementação em sala de aula. São Paulo: Cengage Learning, 2013. Cap. 1, VII, p.1-20.

CAVICHIA ATANAZIO, A. M.; LEITE, Á. E. Tecnologias da Informação e Comunicação (TIC) e a Formação de Professores: Tendências de Pesquisa. Investigações em Ensino de Ciências, Porto Alegre, v. 23, n. 2, p. 88-103, 2018. Disponível em: https://www.if.ufrgs.br/cref/ojs/index.php/ienci/ article/view/947. Acesso em: 20 out. 2019.

DEWEY, J. Vida e educação. São Paulo: Nacional, 1950. 
FERREIRA, N. S. A. As pesquisas denominadas - estado da arte. Educação \& Sociedade, Campinas, v. 23, n.79, p.257-272, ago., 2002. Disponível em: http://www.scielo.br/pdf/es/v23n79/10857.pdf. Acesso em: 20 out. 2019.

FREITAS, R. A. M. M. Ensino por problemas: uma abordagem para o desenvolvimento do aluno. Educação e Pesquisa, São Paulo, v. 38, n.2, p. 403418, abr./jun. 2012. Disponível em: http://www.scielo.br/pdf/ep/v38n2/aop478.pdf. Acesso em: 20 out. 2019.

GAMA RUSSO, A. L. R.; RÔÇAS, G. Analisando as dissertações e teses da área de ensino na perspectiva da história e da filosofia da ciência no ensino de química. Alexandria: Revista de Educação em Ciência e Tecnologia, Florianópolis, v. 12, n. 1, p. 157-180, maio 2019. Disponível em: https://periodicos.ufsc.br/index.php/alexandria/article/view/19825153.2019v12n1p157. Acesso em: 09 nov. 2019.

GIL, A. C. Como elaborar projetos de pesquisa. São Paulo, Atlas, 4.ed, 2002.175p.

KENSKI, V. M. Aprendizagem mediada pela tecnologia. Revista Diálogo Educacional, Curitiba, v. 4, n. 10, p. 47-56, 2003. Disponível em: https://periodicos.pucpr.br/index.php/dialogoeducacional/article/view/6419/63 23. Acesso em: 20 out. 2019.

LACANALLO, L. F.; SILVA, S. S. C.; OLIVEIRA, D. E. M. B; GASPARIN, J. L.; TERUYA, T. $K$. Métodos de ensino e de aprendizagem: uma análise histórica e educacional do trabalho didático. In: VII JORNADA DO HISTEDBR - O trabalho didático na história da educação. Atas do Evento, Campo Grande. 2007. Disponível em: http://www.histedbr.fe.unicamp.br/acer_histedbr/jornada/jornada7/_GT4\%20P DF/M\%C9TODOS\%20DE\%20ENSINO\%20E\%20DE\%20APRENDIZAGEM\%20UMA\%2 OAN\%C1LISE\%20HIST\%D3RICA.pdf. Acesso em: 20 out. 2019.

MAZUR, E. Peer Instruction: a revolução da aprendizagem ativa. Tradução: Anatólio Laschuk. Porto Alegre, Penso, 2015. 252 p.

MINAYO, M. C. S. O desafio da pesquisa social. In: MINAYO, M. C. S. (Org.). Pesquisa social: teoria, método e criatividade. Petrópolis, RJ, Vozes. 28. Ed. 2009. 108 p. Cap.1, p.9-30.

MORAN, J. Mudando a educação com metodologias ativas. Coleção Mídias Contemporâneas - Convergências Midiáticas, Educação e Cidadania: aproximações jovens, v. 2, p. 15-33, 2015.

MORAN, J. Metodologias ativas para uma aprendizagem mais profunda. In: BACICH, L.; MORAN, J. (Orgs.). Metodologias ativas para uma educação inovadora: uma abordagem teórico-prática. Porto Alegre: Penso, 2018. 238 p. Parte I, p.2-25.

MOTOKANE, M. T. Sequências didáticas investigativas e argumentação no ensino de ecologia. Ensaio - Pesquisa em Educação em Ciências, Belo Horizonte, v.17, n.especial, p. 115-137, nov. 2015. Disponível em: 
http://www.scielo.br/pdf/epec/v17nspe/1983-2117-epec-17-0s-00115.pdf. Acesso em: 20 out. 2019.

MÜLLER, M. G.; ARAUJO, I. S.; VEIT, E. A.; SCHELL, J. Uma revisão da literatura acerca da implementação da metodologia interativa de ensino Peer Instruction (1991 a 2015). Revista Brasileira de Ensino de Física, São Paulo, vol. 39, n. 3 , e3403, 2017. Disponível em: http://www.scielo.br/pdf/rbef/v39n3/1806-1117rbef-39-03-e3403.pdf. Acesso em: 20 out. 2019.

NASCIMENTO, T. E.; COUTINHO, C. Metodologias ativas de aprendizagem e o ensino de Ciências. Multiciência, Universidade Regional Integrada do Alto Uruguai e das Missões, Santiago, p.134-153. 2016. Disponível em: http://urisantiago.br/multicienciaonline/adm/upload/v2/n3/7a8f7a1e21d061000 1959f0863ce52d2.pdf. Acesso em: 20 out. 2019.

QUEIROZ, S. L.; CABRAL, P. F. de O. (Orgs.). Estudos de caso no ensino de ciências naturais. São Carlos, Art Point, 2016. 116 p. Disponível em: http://200.144.244.96/livros/2016-Estudos_de_Caso.pdf. Acesso em: 20 out. 2019.

ROMANOWSKI, J. P.; ENS, R. T. As pesquisas denominadas do tipo "Estado da Arte". Revista Diálogo Educacional, Paraná, v. 6, n. 19, 2006. Disponível em: https://www.redalyc.org/pdf/1891/189116275004.pdf. Acesso em: 20 out. 2019.

SASSERON, L. H. Alfabetização científica, ensino por investigação e argumentação: relações entre ciências da natureza e escola. Ensaio - Pesquisa em Educação em Ciências, Belo Horizonte, v. 17, n. especial, p. 49-67, nov., 2015. Disponível em: http://www.scielo.br/pdf/epec/v17nspe/1983-2117-epec-17-0s00049.pdf. Acesso em: 20 out. 2019.

SASSERON, L. H.; CARVALHO, A. M. P. Construindo argumentação na sala de aula: a presença do ciclo argumentativo, os indicadores de alfabetização científica e o padrão de Toulmin. Ciência \& Educação, Bauru, v. 17, n. 1, p. 97-114, 2011. Disponível em: http://www.scielo.br/pdf/ciedu/v17n1/07. pdf. Acesso em: 20 out. 2019.

SILVA, E. T. Os (des)caminhos da escola: traumatismos educacionais. Barafunda metodológica, cap. 1, p. 24-34, Cortez Editora. 104 p. 2011.

SILVEIRA, D. T.; CÓRDOVA, F. P. A pesquisa científica. In: GERHARDT, E.; SILVEIRA, D. T. (Org.). Métodos de pesquisa. Porto Alegre, editora da UFRGS, 2009. 120 p. Unidade 2, 31-42. Disponível em: http://www.ufrgs.br/cursopgdr/downloadsSerie/derad005.pdf. Acesso em: 20 out. 2019.

SOUZA, S. C.; DOURADO, L. Aprendizagem baseada em problemas (ABP): um método de aprendizagem inovador para o ensino educativo. Holos, Rio Grande do Norte, v. 5, ano 31, p. 182-200, 2015. Disponível em: http://www2.ifrn.edu.br/ojs/index.php/HOLOS/article/view/2880/1143. Acesso em: 20 out. 2019. 
TRIVELATO, S. L. F.; TONIDANDEL, S. M. R. Ensino por investigação: eixos organizadores para sequências de ensino de biologia. Ensaio - Pesquisa em Educação em Ciências, Belo Horizonte, v. 17, n. especial, p. 97-114, nov., 2015. Disponível em: http://www.scielo.br/pdf/epec/v17nspe/1983-2117-epec-17-0s00097.pdf. Acesso em: 20 out. 2019.

VALENTE, J. A. Blended learning e as mudanças no ensino superior: a proposta da sala de aula invertida. Educar em Revista, Curitiba, Brasil, Editora UFPR, s/v., edição especial n. 4, p. 79-97, 2014a. Disponível em:

http://www.scielo.br/pdf/er/nspe4/0101-4358-er-esp-04-00079.pdf. Acesso em: 20 out. 2019.

VALENTE, J. A. A comunicação e a educação baseada no uso das tecnologias digitais de informação e comunicação. Unifeso - Humanas e Sociais, v. 1, n. 01, p. 141-166, 2014b. Disponível em:

http://www.revista.unifeso.edu.br/index.php/revistaunifesohumanasesociais/art icle/view/17/24. Acesso em: 20 out. 2019.

VALENTE, J. A.; BARANAUSKAS, M. C. C.; MARTINS, M. C. (Orgs.) ABInv Aprendizagem baseada na investigação. Campinas, SP, UNICAMP, 2014. 371 p.

VALENTE, J. A. A sala de aula invertida e a possibilidade do ensino personalizado: uma experiência com a graduação em midialogia. In: BACICH, L.; MORAN, J. (Orgs.). Metodologias ativas para uma educação inovadora: uma abordagem teórico-prática. Porto Alegre, Penso, 2018. 238 p. Cap. 1, p. 26-44.

VALÉRIO, M.; BELETI Junior, C. R. Caracterização da produção acadêmica brasileira sobre a sala de aula invertida. ACTIO, Curitiba, v. 4, n. 3, p. 17-34, set./dez. 2019. Disponível em: https://periodicos.utfpr.edu.br/actio/ article/view/10163. Acesso em: 20 mar. 2020.

ZOMPERO, A. F.; LABURÚ, C. E. Atividades investigativas no ensino de Ciências: aspectos históricos e diferentes abordagens. Ensaio - Pesquisa em Educação em Ciências, Belo Horizonte, v. 13, n. 03. p. 67-80, set./dez., 2011. Disponível em: http://www.scielo.br/pdf/epec/v13n3/1983-2117-epec-13-03-00067.pdf. Acesso em: 20 out. 2019.

Recibido: 19 nov. 2019

Aprobado: 22 abr. 2020

DOI: 10.3895/actio.v5n2.11296

Cómo citar:

SIMÕES, R. C. M.; GAMA RUSSO, A. L. R.; BRAGA, E. S. O.; RÔÇAS, G. Metodologías activas en la enseñanza de las Ciencias: revisión y análisis de publicaciones en revistas del Área Enseñanza en la década de 2008 a 2018. ACTIO, Curitiba, v. 5, n. 2, p. 1-24, mai./ago. 2020. Disponible en:

$<$ https://periodicos.utfpr.edu.br/actio>. Acesso em: XXX

Dirección de envio:

Roberta Cristina Moreira Simões

Rua Lúcio Tavares, n. 1045, Centro, Nilópolis, Rio de Janeiro, Brasil.

Copyright: Este artículo está licenciado bajo los términos de la Licencia Internacional Creative Commons-

Atribución.

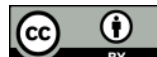

